\title{
A HYPOTHETICAL BURNING-VELOCITY FORMULA FOR VERY LEAN HYDROGEN-AIR MIXTURES
}

\author{
by \\ Forman A. Williams \\ Department of Mechanical and Aerospace Engineering \\ University of California, San Diego \\ La Jolla, CA, 92093 \\ faw@ucsd.edu \\ and \\ Joseph F. Grcar \\ Center for Computational Science and Engineering \\ Lawrence Berkeley National Laboratory \\ Berkeley, CA 94720-8142 \\ jfgrcar@lbl.gov
}

\begin{abstract}
Very lean hydrogen-air mixtures experience strong diffusive-thermal types of cellular instabilities that tend to increase the laminar burning velocity above the value that applies to steady, planar laminar flames that are homogeneous in transverse directions. Flame balls constitute an extreme limit of evolution of cellular flames. To account qualitatively for the ultimate effect of diffusive-thermal instability, a model is proposed in which the flame is a steadily propagating, planar, hexagonal, close-packed array of flame balls, each burning as if it were an isolated, stationary, ideal flame ball in an infinite, quiescent atmosphere. An expression for the laminar burning velocity is derived from this model, which theoretically may provide an upper limit for the experimental burning velocity.
\end{abstract}




\section{INTRODUCTION}

With increasing emphasis being given to enhanced future usage of hydrogencontaining fuels in both automotive and stationary-power applications, there is renewed concern about resolving discrepancies between theory and experiment in hydrogen combustion [1]. One glaring discrepancy is that for lean hydrogen-air flames, measured laminar burning velocities significantly exceed those calculated theoretically [2]. This may be seen most clearly in Fig. 1, where the predictions of the San Diego mechanism are compared with the most recent accurate experiments [3-6]. Although the computations, described previously [2], are based on one particular chemical-kinetic mechanism, with other mechanisms the direction of the discrepancies is the same, and the magnitudes of the discrepancies are comparable or larger. Estimated uncertainties in chemical-kinetic and transport parameters are less than would be required to explain the differences. Other possible reasons for the disagreement therefore are worthy of consideration.

Because of the high diffusion coefficient of molecular hydrogen, lean hydrogen-air flames are known to experience diffusive-thermal (cellular) instability [7]. Under very lean conditions, where steady, planar, one-dimensional processes would lead to flame temperatures too low to support combustion, the highly diffusing fuel can migrate preferentially to spots that consequently effectively act as if there were higher fuel concentrations and therefore support higher temperatures, where the resulting fuel sink encourages continued inward fuel diffusion, establishing a persistent nonplanar pattern. Because of the higher effective flame temperature in these reacting cells, the propagation velocity is larger than would be predicted by a purely one-dimensional analysis. The experimental burning velocities therefore may be larger than calculated because of the 
cellular-flame enhancement not permitted by the one-dimensional codes employed, and the consequent extension of the lean flammability limit can be of concern in fire safety.

Corrections for this nonplanar preferential diffusion will be small near stoichiometric conditions but will increase as the mixture is made leaner. In extremely lean mixtures, relatively few and widely spaced reacting cells may be expected to be encountered. An ultimate limiting situation, in the absence of forced or natural convection, could be a single, nonpropagating, stationary, isolated flame ball. In ideal flame balls [8], the flame temperature exceeds the adiabatic flame temperature by an amount determined by the fact that the temperature increase above ambient is that for the planar one-dimensional flame divided by the Lewis number. With a Lewis number of hydrogen of 0.3 , the ultimate temperature increase is larger by a factor of about 3, and if, for example, the flammability limit corresponded to a fixed adiabatic flame temperature (say in the vicinity of a crossover temperature of about $1000 \mathrm{~K}$ at $1 \mathrm{~atm})$, then the flame ball could support that temperature at an initial fuel concentration less than $40 \%$ of that of the planar flame. Near this limit, the propagating laminar flame cannot be a continuous planar front but may be capable of being modeled well by a planar array of flame balls, each of which can be approximated as an isolated ideal flame ball. The intent of the present work is to explore such a model for the steadily propagating laminar flame and to derive a corresponding burning-velocity formula that may then apply under these very lean conditions at which the planar, one-dimensional flame would be well beyond its flammability limit.

There have been many observations and measurements of flame balls in lean hydrogen mixtures [9], and calculations of their structures have been performed with radiative transfer and detailed molecular transport and chemistry included [10,11]. Their 
use in approximations for propagating flames would, of course, be limited to very lean conditions where well-developed cells occur. Such cells were first observed in lean hydrogen systems in 1914 by Coward and Brinsley [12], and more detailed measurements were made later, e.g. $[13,14]$, as useful reviews $[15,16]$ describe in greater detail. The papers reporting the burning-velocity measurements specifically comment on observed cell formation for spherical flames at sufficiently lean conditions $[3,5,6]$, but the flames in the steady counterflow experiment were said to be very flat [4] and yet produced velocities in agreement with the other measurements. Although the strain in the counterflow is known to suppress visible cells, it nevertheless may not be sufficient to eliminate transverse preferential diffusion that may result in similar burning velocities. The present model may not apply to the counterflow because of the strain, but it may approximate a limit for effectively overall planar freely propagating flames in the absence of disturbances ( such as mean curvature effects at smaller radii in spherical or cylindrical configurations, walls, convection, shear, turbulence, cell interactions or gravity, for example, although these could be considered in later investigations).

\section{THE CHEMISTRY}

The detailed chemistry of hydrogen-air combustion is now well known [2]. The chain-branching step $\mathrm{H}+\mathrm{O}_{2} \rightarrow \mathrm{OH}+\mathrm{O}$ is of paramount importance, and the chaintermination step $\mathrm{H}+\mathrm{O}_{2}+\mathrm{M} \rightarrow \mathrm{HO}_{2}+\mathrm{M}$ competes with it and also leads to most of the heat release. The rates of these two steps are equal at a crossover temperature (approximately $1000 \mathrm{~K}$ at $1 \mathrm{~atm}$ ), and below that temperature the branching rate is slower than the termination rate, so that a branched-chain explosion cannot occur. This causes the 
overall reaction rate to be much lower in deflagrations at initial fuel concentrations that produce adiabatic flame temperatures below crossover, resulting in calculated laminar burning velocities for steady, planar, adiabatic flames that are much lower. These lower burning velocities lead to relatively enhanced effects of heat loss, such as transparent-gas radiation, and therefore flammability limits can be viewed as corresponding approximately to conditions at which the adiabatic flame temperature is below crossover.

Systematic reduction of this chemistry leads ultimately to a two-step reducedchemistry description that can be used to calculate laminar burning velocities through rateratio asymptotics (RRA) [17]. For very lean flames, an accurate one-step approximation to this two-step description has recently been found [18]. The same types of RRA approaches could be used to describe the structures of flame balls (although this has not yet been done). The enhancement in the flame temperature through the Lewis-number effect would be important in such descriptions (and has been obtained in hydrogen-air flame-ball computations with detailed chemistry $[10,11])$. It is simpler, however, in investigating flame-ball modes to use a one-step chemistry model with high activation energy and then to apply activation-energy asymptotics (AEA), as has been done for planar deflagrations [7] (as well as for most of the original flame-ball analyses). The result may be expected to yield the same type of behavior as that which would be obtained by RRA because it has been shown that RRA leads to burning-velocity predictions in agreement with those of AEA when an effective AEA activation temperature equal to four times the square of the maximum flame temperature divided by the difference between the maximum flame temperature and the crossover temperature is used $[17,19]$, reflecting the strong variation in the overall activation energy as crossover is approached. In the present work, to be able to 
use results of existing flame-ball analyses directly, the chemistry that is employed is onestep AEA. This also has the advantage of enabling comparisons with previous results for planar flames [7] to be made readily. Analyses by RRA, tying the predictions more clearly and directly to the underlying detailed chemistry, are left for future investigations.

\section{THE IDEAL FLAME BALL}

Numerous analyses of flame-ball structures have now been published, addressing many different aspects of their structures and dynamics [20-24]. Although the simplest ideal adiabatic flame ball is unstable, radiant energy loss from the hot zone, for example, can introduce a stable branch in the solution [21]. Analyses have been completed for effects of small convection, walls, other nearby flame balls, etc., showing, for example, that flame balls tend to repel each other [22]. Many of these aspects are relevant to the present application since convection will be present in the propagating flame, and mutual flameball interactions will occur. Although modifications to flame-ball structures and motions associated with radiant energy loss, convection and interactions need to be considered in the present problem, as a first step, to obtain the simplest possible formulation, these effects will be ignored, and stability questions will not be addressed. Since radiant loss increases flame-ball sizes, and repulsion tends to disperse flame balls, the results will produce denser flame-ball concentrations than actually will occur, leading to an upper bound for the burning velocity.

Let $\mathrm{D}$ represent the diffusion coefficient of the fuel and $\alpha$ the thermal diffusivity. The Lewis number is then

$$
\mathrm{L}=\alpha / \mathrm{D}
$$


If $\mathrm{T}$ denotes temperature, $\mathrm{Y}$ fuel mass fraction, $\mathrm{Q}$ the heat released per unit mass of fuel consumed and $\mathrm{c}_{\mathrm{p}}$ the specific heat at constant pressure for the mixture (assumed constant), and if subscripts $u$ and $f$ identify unburnt conditions and conditions at the reaction zone of the flame, respectively, then the adiabatic flame temperature is $T_{a f}=T_{u}+Q Y_{u} / c_{p}$, but the temperature in the interior of the ideal flame ball is [23, Eqn. 5]

$$
T_{\mathrm{f}}=\mathrm{T}_{\mathrm{u}}+\mathrm{QY} \mathrm{u} /\left(\mathrm{c}_{\mathrm{p}} \mathrm{L}\right),
$$

which exceeds $T_{a f}$ for $L<1$. With the mass rate of consumption of fuel expressed as $\rho \mathrm{YAe}^{-\mathrm{T}_{\mathrm{a}} \mathrm{T}}$, where $\rho$ denotes density (assumed constant) and $\mathrm{T}_{\mathrm{a}}$ the activation temperature, it follows readily from the previously cited literature that the radius of an ideal flame ball is

$$
\mathrm{R}=\left(\frac{\mathrm{T}_{\mathrm{a}} \mathrm{QY} \mathrm{Y}_{\mathrm{u}}}{\mathrm{c}_{\mathrm{p}} \mathrm{L}^{2} \mathrm{~T}_{\mathrm{f}}^{2}}\right) \sqrt{\frac{\mathrm{D}}{2 \mathrm{Ae}^{-\mathrm{T}_{\mathrm{a}} / \mathrm{T}_{\mathrm{f}}}}},
$$

and the total mass rate of consumption of fuel by the flame ball is

$$
\dot{\mathrm{m}}=4 \pi \mathrm{R} \rho \mathrm{DY} \mathrm{u}_{\mathrm{u}} .
$$

These are the results for the ideal flame ball that are used in the following model.

\section{THE MODEL FOR FLAME PROPAGATION}

Let us assume that, instead of occurring at a continuous reaction sheet, to leading order in AEA the reaction occurs at the surfaces of an array of identical ideal flame balls. For a steadily propagating deflagration, the mass rate of consumption of fuel per unit area is $Y_{u} \rho v$, where $v$ denotes the laminar burning velocity. Let us hypothesize that there is a uniform planar array of flame balls, the number per unit area being $1 / \ell^{2}$, where $\ell$ is a measure of the average distance between the centers of the flame balls. Then the equation for mass conservation of the fuel can be written as 


$$
\mathrm{Y}_{\mathrm{u}} \rho \mathrm{v}=\dot{\mathrm{m}} / \ell^{2}
$$

With this model there must exist a downstream zone in which the nonuniform temperature field relaxes to the adiabatic flame temperature, but this zone is not relevant to the propagation velocity and therefore is not analyzed here. Use of Eq. 4 in Eq. 5 gives, for the laminar burning velocity,

$$
\mathrm{v}=4 \pi \mathrm{RD} / \ell^{2}
$$

Substitution of Eq. 3 into this expression would provide an expression that could be used to calculate $\mathrm{v}$ if the spacing $\ell$ were known. According to this result, the burning velocity varies inversely as the square of the spacing $\ell$. At this level, the result should apply in general for any propagation mode involving complete consumption of fuel by diffusion to highly dispersed spherical sinks of radius $\mathrm{R}$, separated by distances of order $\ell$. Although an analysis of flame-ball dynamics would be needed to determine $\ell$, an upper bound for $\mathrm{v}$ can be obtained from the present result.

The distance between the centers of flame balls in a plane must exceed $2 \mathrm{R}$, or else they would intersect. The densest pattern of spheres in a plane that is possible is the hexagonal (also called triangular) close-packed array, for which

$$
\ell^{2}=2 \sqrt{3} R^{2}
$$

There is some computational evidence for hexagonal patterns in cellular flames, such as Fig. 26 of the review by Kadowaki and Hasegawa [25]. Of course flame balls would not behave as isolated flame balls at such close packing, but since their interactions reduce $\dot{\mathrm{m}}$, use of the isolated ideal flame-ball results with the assumption of a hexagonal close-packed array still yields an upper bound for v. Although nonplanar arrays, such as close-packed 
tetrahedral arrangements, could give even greater rates of overall fuel consumption per unit area, it seems quite unlikely that consumption rates greater than that of the planar hexagonal close-packed array could occur in reality. As an upper-bound estimate, Eq. 7 will therefore be substituted into Eq. 6, giving

$$
\mathrm{v}=\frac{2 \pi \mathrm{D}}{\sqrt{3} \mathrm{R}}=\frac{2 \pi \mathrm{Q} Y_{\mathrm{u}}}{\mathrm{c}_{\mathrm{p}} \mathrm{T}_{\mathrm{a}}}\left(1+\frac{\alpha \mathrm{c}_{\mathrm{p}} \mathrm{T}_{\mathrm{u}}}{\mathrm{DQ} \mathrm{Y}_{\mathrm{u}}}\right)^{2} \sqrt{\frac{2}{3} \mathrm{DAe}^{-\mathrm{T}_{\mathrm{a}} / \mathrm{T}_{\mathrm{f}}}},
$$

in which Eqs. 1, 2 and 3 have been used in writing the final expression. Equation 2 is to be employed for $\mathrm{T}_{\mathrm{f}}$ in the Arrhenius factor in Eq. 8.

\section{COMPARISONS WITH OTHER RESULTS}

It is of interest to compare Eq. 8 with the classical leading-order AEA burningvelocity formula obtained with the same chemistry [7], namely,

$$
\mathrm{v}=\frac{\mathrm{QY}_{\mathrm{u}}}{\mathrm{c}_{\mathrm{p}} \mathrm{T}_{\mathrm{a}}}\left(1+\frac{\mathrm{c}_{\mathrm{p}} \mathrm{T}_{\mathrm{u}}}{\mathrm{QY} Y_{\mathrm{u}}}\right)^{2} \sqrt{2 \frac{\alpha^{2}}{\mathrm{D}} \mathrm{Ae}^{-\mathrm{T}_{\mathrm{a}} / \mathrm{T}_{\mathrm{af}}}} .
$$

The largest difference is the appearance in Eq. 8 of $\mathrm{T}_{\mathrm{f}}$, determined by Eq. 2, in place of $\mathrm{T}_{\mathrm{af}}$ in the Arrhenius factor. For $\mathrm{L}<1(\alpha<\mathrm{D})$ this leads to a higher burning velocity, tending to reduce the differences seen in Fig. 1. This effect is mitigated by the squared factor preceding the square root, but only slightly, the additive terms proportional to $T_{u}$ being small, typically less than 0.1 . The only other difference, aside from a constant factor of $2 \pi / \sqrt{3}$, is the appearance of $\mathrm{D}$ in Eq. 8 rather than $\alpha^{2} / \mathrm{D}$, as the relevant diffusivity inside the square root. This is understandable because the rate of transport of fuel into a flame ball increases in proportion to the fuel diffusivity, while increasing the fuel diffusivity in an ordinary flame decreases the burning velocity because it decreases the 
average fuel concentration in the reaction zone by the factor $\alpha / \mathrm{D}$ and thereby decreases the reaction rate when it is proportional to the fuel concentration [26]. In the familiar idea that the burning velocity is proportional to the square root of the product of the thermal diffusivity and the reaction rate, the fuel diffusivity replaces the thermal diffusivity in the present model because that is what controls the transport in the flame-ball mechanism. Equation 8 thus helps in many ways to improve understanding of how preferential fuel diffusion in lean mixtures at low Lewis numbers enhances the laminar burning velocity.

An attempt may be made to apply Eq. 8 numerically to hydrogen by introducing the RRA result

$$
\mathrm{T}_{\mathrm{a}}=4 \mathrm{~T}_{\mathrm{f}}^{2} /\left(\mathrm{T}_{\mathrm{f}}-\mathrm{T}^{0}\right)
$$

where $\mathrm{T}^{0}$ denotes the crossover temperature, and evaluating A by fitting the experimental burning velocity at a chosen lean equivalence ratio. Because of the variation of the effective overall activation energy with equivalence ratio, this approach should be better than selecting a constant value of $\mathrm{T}_{\mathrm{a}}$. When this is done, with fit points chosen at equivalence ratios of 0.5 and 0.7 , for example, the variation of the burning velocity with the equivalence ratio is slightly weaker than experiment when Eq. 8 is employed but much stronger than experiment when Eq. 9 is employed. This suggests that, although fully ideal flame balls are not formed under these experimental conditions, the tendency to form them is greater than the tendency to maintain transversely homogeneous, continuous planar flame fronts. A representative comparison of slopes is shown in Fig. 2, in which the Arrhenius factor, with exponent

$$
-\frac{\mathrm{T}_{\mathrm{a}}}{2 \mathrm{~T}_{\mathrm{f}}}=-\frac{2}{1-\left(\mathrm{T}^{0} / \mathrm{T}_{\mathrm{f}}\right)}
$$


according to Eq. 10, is the principal cause of the difference, $\mathrm{T}_{\mathrm{f}}$ being given by Eq. 2 for Eq. 8 and by Eq. 2 with $\mathrm{L}=1$ for Eq. 9 . There thus is a reason to prefer Eq. 8 to Eq. 9 for this data, although the true situation lies in between. A proper full RRA flame-ball analysis would provide a much better test for lean hydrogen flames, and future work also should include Soret effects, which modify predictions of Eq. 8 significantly by further increasing the flame-ball temperature. Direct numerical simulation also can help.

\section{DIRECT NUMERICAL SIMULATION}

That a premixed flame front can propagate in the form of flame balls when the mixture is leaner than the flammability limit for planar flames may be demonstrated by direct numerical simulation. The ideal software for this purpose is that described by Day and Bell [27], based on the low-Mach-number formulation of the reacting-flow equations of Rehm and Baum [28], for which the Courant-Fredericks-Levy condition [29] does not require the very small time steps that would be needed for numerical stability in the presence of acoustic waves. Thus the dynamics of the fronts can be observed over long durations. This software is the product of the mathematical and computational research reported in [27, 30-32] and references therein. Calculations made with this methodology compare well with turbulent flame experiments $[33,34]$

The calculations reported here consider a hydrogen-air mixture with equivalence ratio $\phi=0.2$ (volume of fuel $7.75 \%$ ) which does not appear to support a planar flame at standard temperatures and pressures. The percentage of fuel is above the listed [35] flammability limit (4\%) but between the values (4\% and 9\%) reported (e. g. [15], p.10) for upwardly and downwardly propagating, non-planar flames. The CHEMKIN-PREMIX 
software [36] fails to calculate laminar flame speeds for many hydrogen reaction mechanisms when the mixtures contain less than $11 \%$ fuel $(\phi=0.3)$. Steady planar deflagrations that may exist mathematically at much leaner conditions, for example with flame temperatures below crossover, would propagate very slowly and hardly could occur in the laboratory, being extinguished by radiant loss. The chemical mechanism employed in the present calculations is the hydrogen-oxygen sub-mechanism of GRI-Mech 2.11 [37], which is selected here because of its wide use, although newer mechanisms are available $[2]$.

The direct numerical simulations are performed in two spatial dimensions for rectangular domains with inflow boundary conditions at the bottom, outflow at the top, and periodic conditions at the left and right. Dufour and Soret effects, gravity and radiative losses are neglected. The calculations begin with a $\phi=0.3$ mixture in the form of an artificially wrinkled, continuous flame extending across the width of the channel. A $\phi=0.2$ mixture lies immediately upstream from the flame and flows in at the bottom boundary. The inflow velocity is automatically adjusted to hold the flame approximately $2.5 \mathrm{~cm}$ above the bottom boundary by the control method described in [38]. This standoff distance is about the same, $2 \mathrm{~cm}$, that is used with the standard CHEMKIN-PREMIX software to compute laminar flame speeds (see the freely propagating flame example in [36]). Therefore it is expected the flames have no interaction with the inflow boundary.

Calculations of at least 10 model seconds duration were performed for channels of widths $3.0,4.5,6.0$, and $7.5 \mathrm{~cm}$. The initially continuous flame fronts quickly break into disconnected flames that are convex toward the fresh gas and are separated by narrow, 
extinguished regions. These cells compete for access to upstream fuel and may extinguish, but division of the survivors typically replaces lost cells. In very lean conditions, the spacing between cells appears to be comparable to the cell diameters, and the calculated cell sizes are comparable with those observed experimentally under various conditions $[13,14]$. The increased access to fuel at the flanks allows the reaction fronts to curve back until they completely surround regions in which there is no appreciable combustion, as may be seen in Fig. 3. The channels of widths 3.0 and $4.5 \mathrm{~cm}$ each support flame balls of diameters approximately half the channel width. These balls steadily progress at speeds of 1.76 and $1.29 \mathrm{~cm} / \mathrm{s}$, respectively. The smaller ball burns more vigorously because the increased diffusive flux, in this case of fuel, to the curved flame surface is roughly proportional to the curvature. At a channel width of $6.0 \mathrm{~cm}$ there is one cell that does not close and exhibits repetitive oscillations, while at $7.5 \mathrm{~cm}$ two flame balls develop and oscillate in size and in upstream position. In this last interesting case, the shadowed ball becomes smaller but then overtakes the leading ball because it burns faster; whether this dynamical process is stable over very long time is unclear. All four cases consume essentially all of the inflowing fuel, the outflow of fuel being at most $0.2 \%$ by volume. Related steadily and stably propagating lean-hydrogen flame cells recently have been predicted computationally and some of their properties have been investigated [39].

These calculations demonstrate that under very lean conditions, deflagrations in hydrogen-air systems can form closed surfaces that propagate unidirectionally and exist for periods comparable to the passing time of at least a few flame thicknesses. The greater fuel flux into the closed surfaces that would be enabled by the Soret effect and by the third 
spatial dimension would be expected to increase the propagating speeds of similarly sized balls and may also permit wider spacing.

\section{CONCLUDING REMARKS}

The model that has been suggested here offers a new perspective on laminar flame propagation in very lean mixtures with highly diffusive fuels. The propagation mechanism is viewed qualitatively as the advancement of a collection of point sinks of fuel into the fresh mixture. Those sinks have been approximated here as a planar array of ideal flame balls. To estimate an upper limit for the propagation velocity, the array was placed in a close-packed, hexagonal pattern. The resulting model describes a limit that may be approached in extremely lean hydrogen-air flames, where measured burning velocities will appreciably exceed calculated burning velocities that are based on one-dimensional codes.

In the model as developed here there is no final fuel leakage through the array of flame balls. If the fuel consumption were incomplete, then since less fuel needs to be burnt the predicted burning velocity would be increased by the factor $\left(1-Y_{\mathrm{f}} / \mathrm{Y}_{\mathrm{u}}\right)^{-1}$, where $\mathrm{Y}_{\mathrm{f}}$ is the final fuel mass fraction far downstream, because the rate of fuel consumption per unit area, $Y_{u} \rho v$, would then be replaced by $\left(Y_{u^{-}} Y_{f}\right) \rho v$, while the fuel consumption in each flame ball would remain complete. Experimentally, as the flammability limit is approached, some of the fuel escapes combustion in the dispersed cellular flames. This, however, may be due to the finite times available in experiments. In an infinite system, an infinite amount of time is available for the fuel to diffuse into the array of flame balls. In addition, the numerical simulations did not reveal any appreciable incomplete combustion. It thus may well be a 
reasonable idealized model to neglect influences of fuel penetration, analogous to the classical burning-velocity formulation in a doubly infinite region.

Accurate testing of the model by experiment would be difficult because of the low velocities and long times required. Buoyancy surely would become important in earthbound experiments and would cause the flame-ball array to tend to rise and extinguish on upper surfaces, if indeed a measurable ideal array could even be established under normal gravity. Coward and Jones [40] remark on "balls of flame that traveled to the top of the vessel". Since buoyancy effects plague experiments, computational approaches would offer more promising avenues for testing predictions. The buoyancy-free computations could begin with a steadily propagating front, very lean, employing detailed hydrogen chemistry (such as the initial computational results reported here), systematically reduced hydrogen chemistry or one-step modeled Arrhenius chemistry, and the system could be followed in time to see to what extent arrays of flame-ball-like structures tend to develop. When such computations produce these types of structures, as in Fig. 3, they shed light on their dynamics and interactions, topic which have not be addressed in the model presented here. There is thus much more interesting research to be done on laminar flame propagation in very lean mixtures of highly diffusive fuels.

\section{ACKNOWLEDGMENT}

FAW is indebted to Guy Joulin and to Antonio Sanchez for their comments on earlier drafts of this paper and to Javier Urzay for help in preparing the manuscript. The work of JFG was supported by the Director, Office of Science, Office of Advanced Scientific Computing Research, of the U. S. Department of Energy under Contract No. DE-AC02-05CH11231. 


\section{REFERENCES}

1. $\quad$ R.W. Shefer, Int. J. Hydrogen Energy 28 (2003) 1131-1141.

2. P. Saxena and F.A. Williams, Combust. Flame 145 (2006) 316-323.

3. D.R. Dowdy, D.B. Smith, S.C. Taylor and A. Williams, Proc. Combust. Inst. 23 (1990) 325-332.

4. F.N. Egolfopoulis and C.K. Law, Proc. Combust. Inst. 23 (1990) 333-340.

5. S.D. Tse, D.L. Zhu and C.K. Law, Proc. Combust. Inst. 28 (2000) 1793-1800.

6. O.C. Kwon and G.M. Faeth, Combust. Flame 124 (2001) 590-610.

7. F.A. Williams, Combustion Theory, 2'nd Edition, Addison-Wesley, Redwood City, CA, 1985, pp. 154-165, 349-365.

8. Y.B. Zel'dovich, in Theory of Combustion and Detonation in Gases, Part 1 (N.N. Semenov, ed.) Izd-Vo AN StSSR, Moscow, 1944, p. 71.

9. P. D. Ronney, Proc. Combust. Inst. 27 (1998) 2485-2506.

10. J. Buckmaster, M. Smooke and V. Giovangigli, Combust Flame 94 (1993) 113-124.

11. M.- S. Wu, P. D. Ronney, R. O. Colantonio and D. M. Vanzandt, Combust. Flame 116 (1999) 387-397.

12. H. F. Coward and F. Brinsley, J. Chem. Soc. 105 (1914) 1859-1866.

13. B. Bregeon, A. S. Gordon and F. A. Williams, Combust. Flame 33 (1978) 33-45.

14. T. Mitani and F. A. Williams, Combust. Flame 39 (1980) 169-190.

15. G. H. Markstein, Nonsteady Flame Propagation, Macmillan, New York, 1964, pp 9-87.

16. M. Hertzberg, Prog. Energy Combust. Sci. 15 (1989) 203-239.

17. K. Seshadri, N. Peters and F.A. Williams, Combust. Flame 96 (1994) 407-427. 
18. A. L. Sanchez, personal communication (2008).

19. K. Seshadri, Proc. Combust. Inst. 26 (1996) 831-846.

20. Y.B. Zel'dovich, G.I. Barenblatt, V.B. Librovich, and G.M. Makhviladze, The Mathematical Theory of Combustion and Explosions, Plenum Press, New York, 1985, pp. 327-331.

21. J.D. Buckmaster, G. Joulin and P.D. Ronney, Combust. Flame 79 (1990) 381-392.

22. J. Buckmaster and P. Ronney, Proc. Combust. Inst. 27 (1998) 2603-2610.

23. Y. D’Angelo and G. Joulin, Combust. Theory Modelling 5 (2001) 1-20.

24. Z. Chen and Y. Ju, Combust. Theory Modelling 11 (2007) 427-453.

25. S. Kadowaki and T. Hasegawa, Prog. Energy Combust. Sci. 31 (2005) 193-241.

26. A. Liñán and F.A. Williams, Fundamental Aspects of Combustion, Oxford University Press, New York, 1993, pp. 24-27.

27. M. S. Day and J. B. Bell, Combust. Theory Modelling 4 (2000) 535-556.

28. R. G. Rehm and H. R. Baum, National Bureau of Standards Journal of Research 83 (1978) 297-308.

29. R. Courant, K. Friedrichs and H. Lewy, Math. Ann. 100 (1928) 32-74.

30. M.J. Berger, and P. Colella, J. Comput. Phys. 82 (1989) 64-84.

31. A.S. Almgren, J.B. Bell, P. Colella, L.H. Howell, and M.L. Welcome, J. Comput. Phys. 142 (1998) 1-46.

32. R.B. Pember, L.H. Howell, J.B. Bell, P. Colella, W.Y. Crutchfield, W.A. Fiveland, and J.P. Jessee, Combust. Sci. Technol. 140 (1998) 123-168. 
33. J.B. Bell, M.S. Day, I.G. Shepherd, M. Johnson, R.K. Cheng, J.F. Grcar, V.E. Beckner, and M.J. Lijewski, Proc. Natl. Acad. Sci. USA 102(29) (2005) 1000610011.

34. J.B. Bell, M.S. Day, J.F. Grcar, M.J. Lijewski, J.F. Driscoll, and S.F. Filatyev, Proc. Combust. Inst. 31 (2007) 1299-1307.

35. I. Glassman, Combustion, 3'rd Edition, Academic Press, San Diego, 1996, p. 585.

36. R.J. Kee, J.F. Grcar, M.D. Smooke and J.A. Miller, PREMIX, Report SAND858240, Sandia National Laboratories, 1985.

37. C.T. Bowman, R.K. Hanson, D.F. Davidson, W.C. Gardiner, Jr., V. Lissianski, G.P. Smith, D.M. Golden, M. Frenklach and M. Goldenberg, available at $<$ http://www.me.berkeley.edu/gri_mech/>.

38. J.B. Bell, M.S. Day, J.F. Grcar and M.J. Lijewski, Comm. Appl. Math. Comput. Sci. $1(2005)$ 29-52.

39. J.F. Grcar, Proc. Combust. Inst. 32, to appear (2008).

40. F. Coward and G.W. Jones Limits of Flammability of Gases and Vapors, Bulletin 503, Bureau of Mines, 1952, p. 15. 


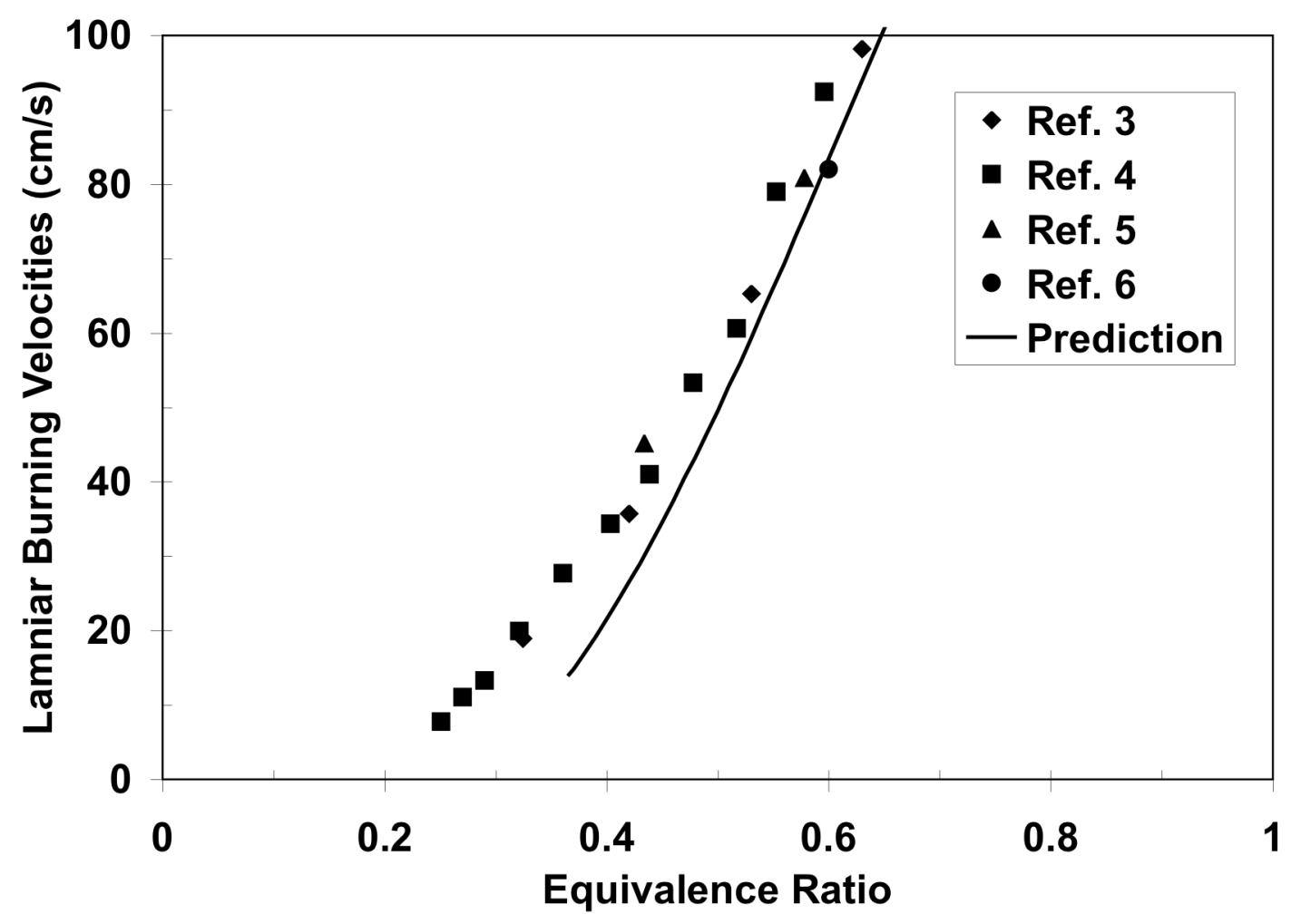

Fig. 1 Comparison of measured and calculated laminar burning velocities of lean hydrogen-air flames at $1 \mathrm{~atm}$ and initially $298 \mathrm{~K}$. 


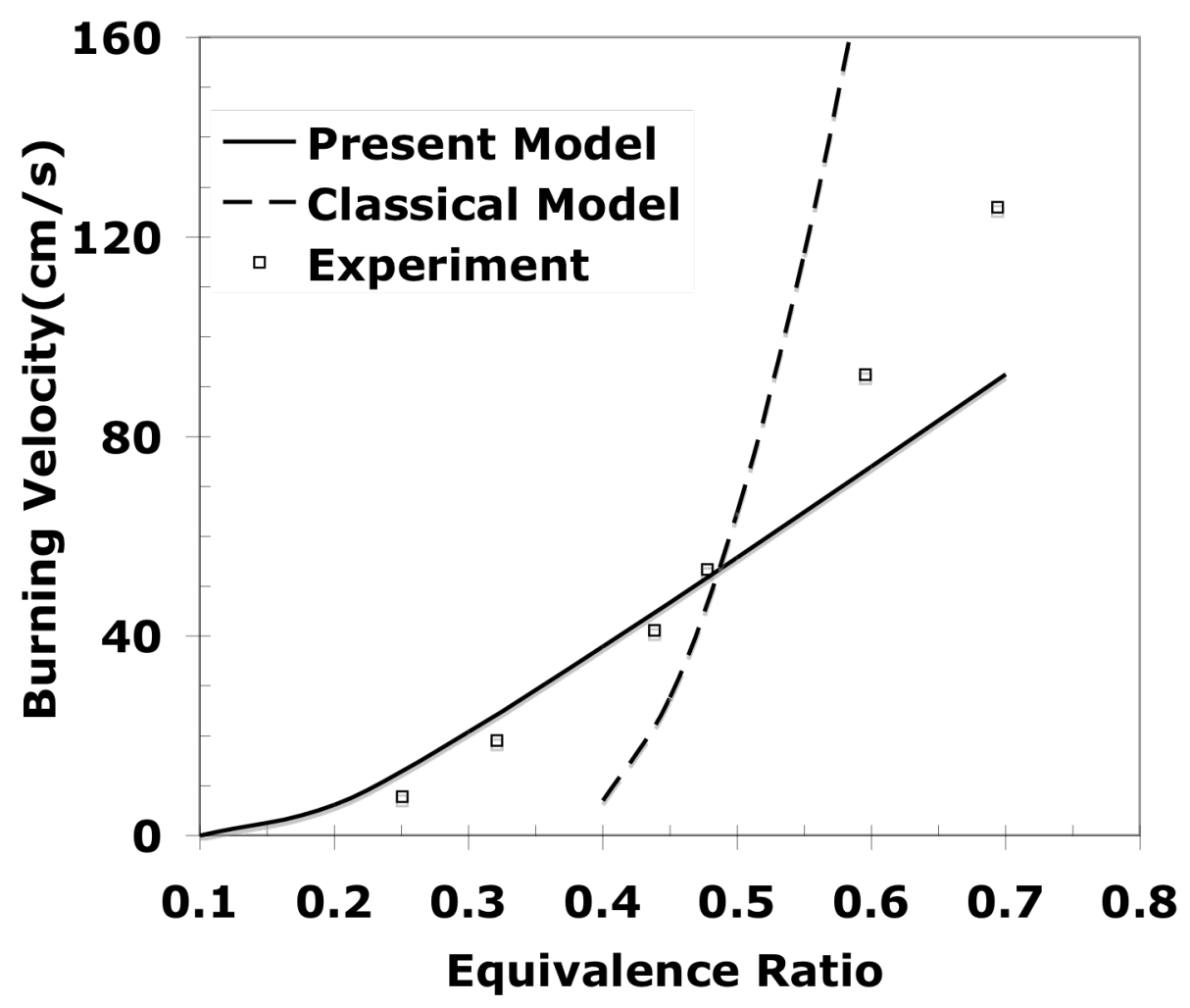

Fig. 2 Comparisons of burning-velocity predictions with experiment, based on AEA with activation energies adjusted according to RRA, for the present model (solid curve) and the classical model (dashed curve), when an experimental burning velocity (points) of $53 \mathrm{~cm} / \mathrm{s}$ is fit at an equivalence ratio of 0.5 . 


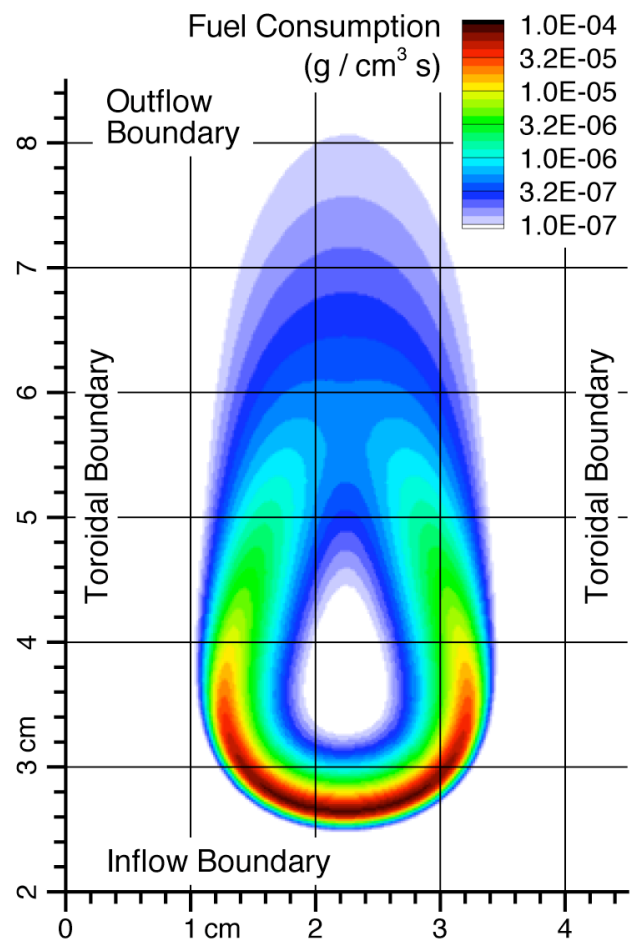

Fig. 3. Fuel consumption in a teardrop-shaped, premixed flame ball that propagates unidirectionally toward inflowing fresh gas. 